der Feststellvorrichtung b nach dem Vorevakuieren) in Betrieb und sorgt durch Offnen des Hahnes X nach u (Stellung , , f̈ $11 t^{\text {") }}$ ) dafür, daß das Quecksilber in dem Druckmesser nicht emporsteigt. Man läßt die Pumpe so lange gehen, bis man an dem harten Anstoßen des Quecksilbers erkennt, daß die Luft schon stark verdünnt ist, und läßt dann einfach durch Drehen des Hahnes $X$ auf ,s t e i g t" das Quecksilber in dem Druckmesser bis zum Ventil w emporsteigen, dadurch wird das Rohr $\beta \gamma \delta s$ völlig mit Quecksilber ausgefüllt. Saugt man nun wieder das Quecksilber nach s zurück (Hahnstellung ,,f ä $\left.11 t^{6}\right)$, so bleibt in dem U-förmigen Teile $\delta \varepsilon$ ein Quecksilberfaden liegen, und indem das Quecksilber bei $\alpha$ abreißt, entsteht über dem im Teile $\gamma$ befindlichen Quecksilberniveau ein absolutes Va. kuum.

Der Teil $\beta \gamma$ ist nunmehr ein abgekürztes Manometer, da über $\gamma$ absolutes Vakuum ist und über $\beta$ der in der Pumpe herrschende Druck lastet.

Eine Spiegelskala hinter $\beta \gamma$ erleichtert die Ablesungen. Die Differenz der Quecksilberhöhen in $\beta$ und $\gamma$ gibt den in dem Apparat herrschenden Gesamtdruck an (wie bei Fig. 2 das mit $A$ bezeichnete abgekürzte Barometer).

Die Ablesungen an diesem Barometer kann man nun auf der ganzen Länge der Skala vornehmen, äußerst tiefes Vakuum nötig ist, welches man wegen der Gummischläuche, welche nicht völlig luftdicht sind, nicht erzielen kann.

Als Vaku u destillationsvorlage ist für manche $Z$ wecke die nachfolgend beschriebene zweckmäßig.

\section{Vakuumdestillationsvorlage mit Quecksilberdichtungen ${ }^{1}$ ).}

\section{Von Leo Ubbelonde.}

Mitteilung aus dem kgl. Materialprüfungs-Amt. (Eingeg. d. 7./3. 1906.)

Je tieferes Vakuum erreicht werden soll, um so größere Ansprüche müssen an die Luftdichtheit der Destillationsgefäße gestellt werden.

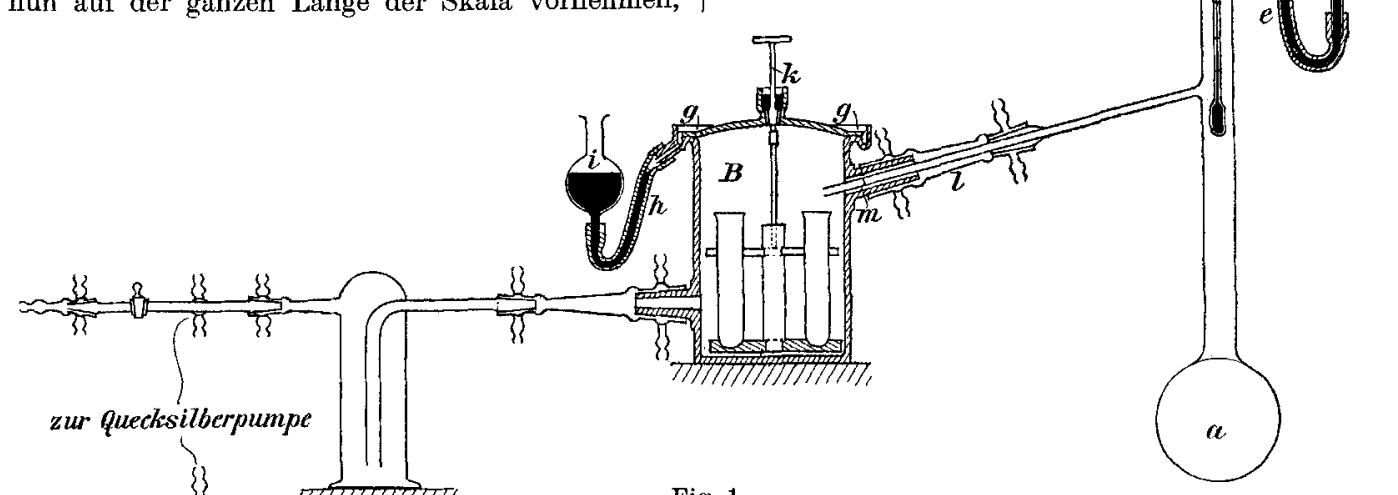

Fig. 1.

Zur Destillation von Mineralölen, Fettsäuren usw. unter sehr tiefem Vakuum wurde bei Durchführung der oben erwähnten Arbeiten die nachfolgend beschriebene Destillationsvorlage kon-

also ebensowohl wenn das Quecksilber unten steht und die Kompressionsdruckmessung außer Tätigkeit ist, als auch wenn das Quecksilber nach oben gestiegen und die Kompressionsdruckmessung in Tätigkeit ist. Damit keine Fehler durch die Wirkungen der Oberflächenspannung entstehen, müssen die Rohre genügend weit und von gleichem Durchmesser sein.

Eine etwas abweichende Konstruktion des zuletzt beschriebenen Apparates zeigt noch Fig. 4.

Die Bewegung des Quccksilbers wird hier ohne Benutzung einor Wasserstrahlpumpe durch Heben und Senken des Gefäßes s bewirkt, welches mittels der Schläuche C und D mit dem Apparat verbunden ist. Die Abkürzung des Druckmessers wurde in diesem Falle in der einfachsten Weise dadurch möglich, da $B$ durch die Schlauchverbindung $D$ über dem Quecksilberniveau in s auch nur der kleine im Rezipienten vorhandene Druck herrscht.

Diese Einrichtung wird nur da zu wählen sein, wo man keine Vorpumpe benutzen will, und wo kein struiert.

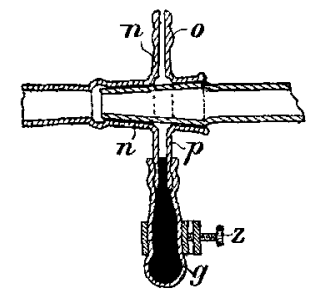

Fig. 2 a.

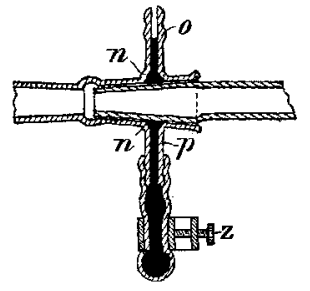

Fig. $2 b$.

Sie hat die Form der B r ü h l schen Vorlage. Sämtliche Verbindungen sind jedoch durch Glasschliffe hergestellt unter Anwendung verschiedener

1) Der Apparat und die Quecksilberverschlüsse sind geschützt. Verfertiger $W$ a r m b r u n, Q u ilitz \& C o., Berlin, Heidestr. und B l e c k m a n n \& Burger, Berlin, Johannisstr. 14/15. 
neuartiger Quecksilberdichtungen, welche sich dadurch auszeichnen, daß sie schnell und einfach zu öffnen und zu schließen sind, ohne daß Quecksilber aus- und eingefüllt werden muß.

Zum Betriebe wird die oben beschriebene Quecksilberpumpe mit abgekürztem Druckmesser für sehr kleine Drucke benutzt. Da die Vorlage duroh die Quecksilberdichtungen vollkommen luftdicht ist, so kann man jeden kleinsten Druck in kurzer Zeit erzeugen und beliebig lange und vollkommen gleichmäßig erhalten.

In dem Halse des Destillationskolbens a (siehe Fig. 1) ist das Thermometer b durch den Gummistopfen $c$ befestigt. Den oberen Teil des Halses umschließt eine Hülse $d$ aus elastischem Gummi, welche noch über das obere Ende des Gummistopfens hinausragt und mittels des Ansatzrohres o mit dem Gefä $B$ f verbunden ist. In dem GefäB $f$ befindet sich Quecksilber, welches durch Heben in die Hülse d fließt, alsdann den Gummistopfen bedeokt und luftdicht abschließt.

In ähnlicher Weise ist die Quecksilberdichtung des Deckels der Vorlage B ausgeführt. Rings um den Deckelschliff läuft eine Glasrinne $\mathrm{g}$, welche durch den Gummischlauch $h$ mit dem Gefäß $i$ in Verbindung steht. Durch Heben des Gefäßes i tritt Quecksilber in die Rinne ein und schließt dadurch den Schliff luftdicht ab.

Der zum Vordrehen des die einzelnen Auffangegefäße enthaltenden Drehgestells durch den Deckel geführte Stab ist mit einer einfachen Quecksilberdichtung $k$ gedichtet.

Die Verbindung $\mathrm{zwischen}$ der Vorlage $\mathrm{B}$ und dem Destillationskolben a ist mittels des Zwischenstücks 1 hergestellt. Durch einen einzelnen Schliff konnte die Verbindung nicht hergestellt werden, da infolge der plötzlichen Erwärmung bei Bcginn der Destillation der Ansatz $m$ an der aus gegossenem Glase bestehenden Vorlage zu leicht springt.

Die bei dem $Z$ wischenstück I und an den übrigen horizontalen Schliffen angewandte Quecksilberdichtung zeigt in größerem Maßstabe Fig. 2a und $2 \mathrm{~b}$. Der Mantelteil des Schliffes besitzt etwa in der Mitte eine ganz herumlaufende, ringförmige Auswölbung $\mathbf{n}$, die oben und unten mit den beiden Ansatzrohren $o$ und $p$ in Verbindung steht. An das untere Ansatzrohr p ist ein kleines Gummisäckchen q gehängt, welches mit Quecksilber gefüllt ist. Über das Gummisäckchen ist eine einfache Schraubenklemme geschoben. Nach Zusammenstecken des Schliffes wird durch Anziehen der Schraubenklemme der Gummisack zusammengedrückt und dadurch das Quecksilber nach oben gedrückt (Fig. 2b). Aus dem Rohr $p$ tritt es in die ringförmige Wulst $n$, umschließt beim Höhersteigen den Schliff vollständig, bis es schließlich in das Rohr o eintritt. Soll der Schliff geöffnet werden, so läßt man zunächst das Quecksilber durch Zurückschrauben der Klemme wieder in das Säckchen zurïckfließen (Fig. 2a) und kann dann den Schliff öffnen. Gange.

Untersuchungen mit dieser Vorlage sind im
Apparat zur schnellen und

\section{kontinuierlichen Entwicklung von Wasserdampf.}

Von K. Beck.

Mitt. a. d. Labor. f. angew. Chemie der Universität Leipzig. Der neue Dampfentwickler soll dem Bedürfnis abhelfen, schnell und jederzeit einen kontinuierlichen Dampfstrom zur Verfügung zu haben, und im be-

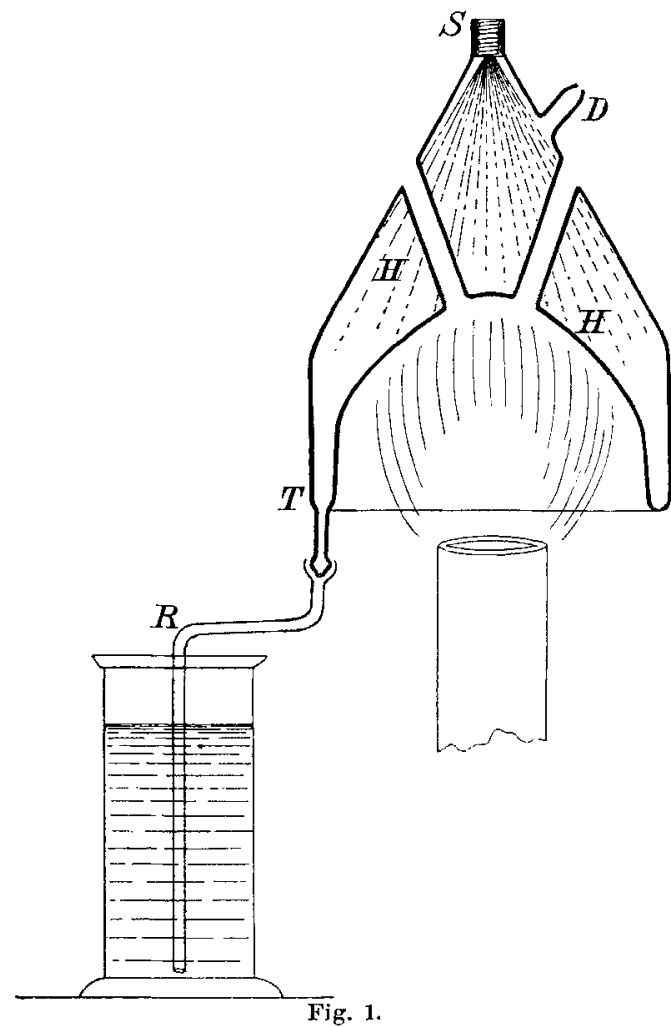

sondern die sonst üblichen Dampfentwickler aus Kupferblech, deren nachteilige Figenschaften sich

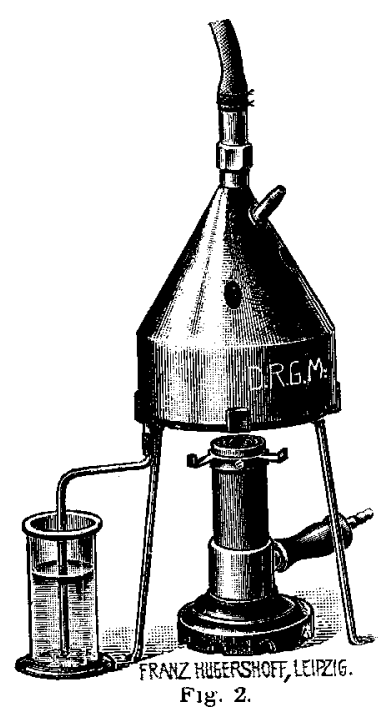

im Laboratoriumsbetrieb bei lebhafter Benutzung oft fühlbar machen, ersetzen.

Der durch die beigefügte Skizze(Fig.1) erläuterte Apparat ist in folgender Weise konstruiert. Das Wasser der Leitung, dem mit Hilfe einer Körtingschen Zerstäubungsdüse $S$ eine große Oberfläche erteilt wird, gelangt in dem geheizten kleinen Kessel H bzw. an dessen Heizflächen zurVerdampfung. Das überschüssige und kondensierte Wasser fließt

beständig durch $\mathrm{T}$ ab. Der Dampf entweicht durch den Tubus $D$. Um zu vermeiden, daß 